\title{
Determinación de Línea Base para el Desarrollo de Actividades Comunitarias de la Escuela de Ingeniería Automotriz de la Universidad Internacional del Ecuador extensión Guayaquil
}

\section{Baseline Determination for the Community Development Activities of the Automotive Engineering School of the International University of Ecuador extension Guayaquil}

\author{
Oscar Stalin Orellana Cruz \\ (D) https://orcid.org/0000-0002-9788-8485 \\ Daniela Alexandra Jerez Mayorga \\ (D) https://orcid.org/0000-0002-4668-4175 \\ Dave Eduardo Saltos Jácome \\ Universidad Internacional del Ecuador
}

Autor para correspondencia: osorellanacr@uide.edu.ec; djerez@uide.edu.ec; dasaltosja@uide.edu.ec

Fecha de recepción: 21 de noviembre de 2020 - Fecha de aceptación: 07 de diciembre de 2020

\section{Resumen}

El objetivo de este estudio es poder determinar la línea base para el desarrollo de actividades de vinculación con la comunidad en el programa denominado Laboratorio Social Chongón 2019 - 2022. El proyecto tiene como objeto incrementar el conocimiento en educación vial y ambiental entorno al uso de motos y tricimotos como medio de transporte a través de la trasferencia de conocimiento de estudiantes de la escuela de ingeniería automotriz de la universidad a los habitantes de los alrededores del Centro de Atención Municipal Integral (CAMI) de Chongón. La metodología aplicada en el estudio fue cuantitativa y se realizó mediante la toma de 40 encuestas a personas del sector por parte de estudiantes y docentes de la universidad. El estudio tuvo un carácter descriptivo no inferencial. Las preguntas fueron dirigidas a indagar sobre condiciones del sector como el nivel de señalización, accidentes (fuentes y concentración geográfica), conocimientos de leyes de tránsito, alcantarillado y servicios básicos. Esta exploración de datos se realizó a una muestra no representativa para la población del sector. Los resultados muestran un alto nivel de accidentes en el centro de Chongón ocasionados por motos donde los encuestados expresan la necesidad de que se mejore la señalética en el sector y capacitaciones que permitan aclarar los requisitos y teoría necesaria para obtener la licencia tipo A, educación vial orientada a la prevención de accidentes y seguridad industrial enfocada en el manejo de residuos. Como conclusión, se determinó la línea base para el desarrollo de actividades de vinculación comunitaria de la Escuela de Ingeniería Automotriz extensión Guayaquil dentro del programa Laboratorio Social UIDE.

Palabras claves: vinculación; línea base; automotriz; educación vial; manejo de residuos; Chongón. 


\begin{abstract}
This study aims to determine the baseline for the development of community activities in the program called Chongón Social Laboratory 2019 - 2022. The goal of the project will be to increase knowledge in road and environmental education around the use of motorcycles and tricycles as conveyance through the transfer of knowledge from students of automotive engineering university's school to the inhabitants of the surroundings of the Center Atenttion Municipal Integral (CAMI) of Chongón. The methodology applied in the study was quantitative and was carried out by taking 40 surveys of people in the sector by students and professors of the university. The study had a non-inferential descriptive character. The questions were directed to inquire about area conditions such as the level of road sign, accidents (sources and geographic concentration), traffic laws knowledge, sewerage and basic services. This data exploration was carried out on a non-representative sample for local residents. The results show a high level of accidents at downtown area caused by motorcycles, where people surveyed express the need to improve road signs and training to answer questions about requirements and knowledge necessary to obtain the type A license, road education oriented to accident prevention and industrial safety focused on waste management. In conclusion, the baseline that is used to prepare community activities of Automotive Engineering School extension Guayaquil within the Social Laboratory UIDE program was determined.
\end{abstract}

Keywords: bonding program; base line; automotive; road safety education; waste management; Chongón.

\title{
Introducción
}

La Ley Orgánica de Educación Superior LOES (2018) estipula en su artículo 13, literal a, que: "Es función del sistema de educación garantizar el derecho a la educación superior mediante la docencia, la investigación y su vinculación con la sociedad, asegurando crecientes niveles de calidad, excelencia académica y pertinencia” (Asamblea Nacional República del Ecuador, 2018, pág. 8). La vinculación debe brindar un aporte a la sociedad por parte de las instituciones académicas, que esté enfocado en grupos vulnerables y que tenga el objetivo de solucionar problemas sociales, problemas ambientales y problemas productivos (Guerrero, y otros, 2019).

Considerando lo antes expuesto, el presente estudio tiene como objetivo determinar una línea base para el desarrollo de actividades de vinculación con la comunidad de la Escuela de Ingeniería Automotriz dentro del programa denominado Laboratorio Social de la Universidad Internacional del Ecuador en el sector de Chongón, reconociendo las condiciones del sector según la percepción de los habitantes respecto a nivel de accidentes y señalización, así como alcantarillado y servicios básicos para identificar necesidades y oportunidades en las que los estudiantes puedan aportar con el conocimiento adquirido en la carrera.

Para el levantamiento de la línea base se aplicarán encuestas y se analizarán los resultados que sirvan de soporte para proponer proyectos orientados a resolver los problemas identificados en el sector mediante capacitaciones en educación vial y ambiental orientadas al incremento en conocimiento por medio de la transferencia de conocimientos de los estudiantes a las personas del sector que tendrá una transformación rural y comunal a urbanizada debido al proyecto de nuevo aeropuerto de Guayaquil anunciado por la Autoridad Aeroportuaria de Guayaquil (AAG), por esta razón es importante capacitar a los habitantes del sector y prestadores de servicio de transporte 
motorizado (motos y tricimotos) en educación vial al ser el principal medio de transporte, además del correcto manejo de residuos en conductores que realicen por su cuenta los mantenimientos para así reducir el riesgo en accidentes de tránsito e impacto ambiental en la zona.

El Aeropuerto estará inmerso en la sociedad, los beneficios del comercio serán cosa del día a día en la nueva ciudad del futuro en Daular, un ejemplo ícono para todo el país, señala la AAG (Autoridad Aeroportuaria de Guayaquil, 2015). Sin embargo, vale recalcar que desde el punto de vista biofísico la comuna San Pedro de Chongón posee un espacio dinámico con una interacción en mosaico de tres sistemas: uno urbano, uno rural y uno natural (Bastidas, P. \& Velasco, E., 2018).

La parroquia urbana Chongón o San Pedro de Chongón, está ubicada al oeste de la ciudad de Guayaquil a la altura del km. 24 de la Autopista Guayaquil-Salinas. Políticamente pertenece al cantón Guayaquil, Provincia del Guayas, y cuenta con una superficie de 1.340 kilómetros cuadrados que significa el 22\% de la superficie del cantón Guayaquil, y con una población de habitantes que bordea las 37.726 personas según el último censo realizado por el Instituto Nacional de Estadísticas y Censos INEC en el año 2010, lo que representa el 1\% de la población cantonal.

Por último, a pesar de que esta parroquia se volvió urbana, la Comuna San Pedro aún cuenta con varias necesidades básicas que afectan las condiciones de vida de sus moradores, tales como alcantarillado, drenaje fluvial, calles y vías pavimentadas, bordes, veredas y demás servicios básicos en los sectores más adentrados de la comuna donde todavía no tienen acceso al agua potable, recolección de basura y energía eléctrica (Mite, S. y Reyes, S., 2018).

\section{Marco teórico}

La guía para establecer investigaciones que señala que, en principio, se debe tener en cuenta que para alinear una investigación se parte del paradigma, luego el enfoque y finalmente se determina el tipo, diseño y método de investigación. (Carhuancho, I., Sicheri, L., Nolazcos, F., Guerrero, M., Casana, K., 2019). El presente trabajo considera el enfoque cualitativo de investigación que, en síntesis, debe tener una etapa inicial exploratoria con el apoyo de las entrevistas y la observación, donde se exponga el contexto del problema, más no aún el problema en sí, sin embargo, el uso de la observación participativa y las entrevistas a profundidad permitirán definir las categorías previas o iniciales del estudio y emergentes; para comprender los eventos con el significado esencial en un marco de estudio profundo de la realidad (Martínez, 2007).

Se establece una investigación exploratoria con la intención de indagar información relevante y trabajos previos que llamen la atención y predisponga su curiosidad analítica para poder empezar con el estudio. Este tipo de investigación se relaciona con el entorno y especialmente a la situación que le inquieta, consulta y revisa referencias bibliográficas surgiendo de este modo una serie de posibilidades que deberá seleccionar para iniciar el proceso de investigación. Por lo tanto, este tipo de investigación no solo abarca el conocimiento previo de los temas a estudiar, sino que direcciona su alcance hacia la justificación especifica de la misma, es te proceso es catalogado como 
la indagación informal libre, debido que permite obtener una agudeza concreta de los aspectos significativos que intervienen en la situación de estudio. (Hurtado de Barrera, 2015).

Izaguirre, Bazurto y Rosado (2020) en su determinación de una línea base del programa de vinculación señalan que el levantamiento de la información cuantitativa se realizó mediante encuestas a diversos negocios del sector por parte de los estudiantes y profesores. El trabajo de campo se basó en recolectar los datos y posteriormente analizar los resultados de las encuestas, las mismas que tuvieron un carácter descriptivo no inferencial, con preguntas orientadas a obtener resultados que permitieron tabular y analizar las características generales y coincidentes de los negocios. A través de los datos que la muestra no representativa brindó, se obtuvo información que permite conocer más a fondo el entorno de la comunidad con la que se pretende trabajar, y obtener por medio de las preguntas planeadas una línea base de la investigación. Frente a esto, se podrán elaborar soluciones para los emprendedores y dueños de negocios que forman parte de la zona.

De acuerdo con los datos del anuario 2019 de la AEADE, desde el 2011 se ha vendido un promedio de 100.974 motos por año, esto representa un incremento de más de 900.000 motos al término del año 2019 al parque automotor nacional. De estas cifras, Guayas representa el 30\%, es decir alrededor de 270.000 motos adicionales al parque automotor que tiene un crecimiento importante a partir del 2016.

Por otro lado, según datos del INEC, existe un total de 582.506 motos matriculadas hasta el 2018, un 25\% menor al número de motos vendidas desde el 2011 al 2018 según los datos de la AEADE en su anuario 2019. El INEC reporta 143.386 motos matriculadas en la provincia del Guayas y esto representa un $37 \%$ menor al número de motos vendidas reportadas por la AEADE en esta provincia en el período 2011-2018.

El número de accidentes de tránsito reportados por el INEC en el 2018 a nivel nacional fue de 25.530 con un total de víctimas de 22.009. Guayaquil representa el 34\% de los accidentes con 8.619 accidentes de los cuales las principales causas son la impericia e imprudencia del conductor (42\%), exceso de velocidad (29\%) y no respetar las señales de tránsito (15\%), estos accidentes en la provincia han dejado un total de 8.330 víctimas con 511 personas fallecidas y las demás lesionadas.

Analizando la serie histórica de siniestros reportados en Guayas por el INEC desde el 2013 al 2018, se tiene una reducción desde el 2013 al 2015 del 35\%, sin embargo, a partir del 2016 esta tendencia comienza a incrementarse, 27\% mayor al finalizar el 2018 frente al 2016 y coincide con el incremento en la venta de motos reportada por la AEADE donde al finalizar el 2018 fue de un 74\% mayor frente al 2016.

De acuerdo al número total de vehículos matriculados en Guayas hasta el 2018, el INEC reporta que por cada 100 siniestros de tránsito 91 personas resultan lesionadas y 6 fallecen, además por cada 1.000 vehículos matriculados se producen 16 siniestros de tránsito. Considerando las cifras de unidades que realizaron la revisión técnica vehicular (RTV) en los centros RTV en la ciudad de Guayaquil del estudio y estimación de emisiones generadas por motos de combustión interna en un sector de alta concentración y análisis de motos eléctricas como una alternativa viable de movilidad (Loaiza, C. \& Gómez, E., 2019), se tienen 38.822 motos revisadas que representan el 96\% del total 
de motos reportadas por el INEC y con ello casi la totalidad de siniestros de tránsito provocados por esta clase se concentran en esta ciudad.

Según el ajuste y revisión del plan maestro de agua potable, alcantarillado sanitario y pluvial de Interagua, corresponde al escenario que incluye, además de la evolución propia del área urbana actual de Guayaquil, el desarrollo del proyecto del Nuevo Aeropuerto de Guayaquil en el área denominada Chongón - Daular, que lleva adelante el Municipio de Guayaquil, y la conclusión del Convenio con Hidroplayas, considerando concurrentemente a largo plazo el desarrollo del Puerto de aguas profundas en Posorja. Asimismo, en virtud que se dispone de los resultados del Censo INEC 2010, se ha efectuado en un ajuste de población actual y su proyección hasta el año 2041. Las diferencias encontradas entre la anterior proyección al año 2010 realizada en base al Censo INEC 2001 y los nuevos datos censales, indica un incremento considerable en el área de Influencia del Nuevo Aeropuerto de la Ciudad de Guayaquil en Daular/Chongón (Interagua). Para el 2021 supone un crecimiento de viviendas en un $323 \%$ y para el 2031 de $1392 \%$.

Para finalizar, el gerente de la Fundación Aeroportuaria de Guayaquil, la institución que inició trabajos en el 2014 como medida de remediación social con la comunidad, señalo, que el sistema de alcantarillado había llegado al $45 \%$ de la población de este sector y que desde septiembre del 2018 para con ello a finales del 2019 el 65\% de la población de Chongón estaría abastecida. El $35 \%$ restante, que corresponde a los hogares de barrio de Colibrí (1,2,3), será construido entre los años 2020 y 2021 (Diario Expreso, 2019).

\section{Metodología}

El levantamiento de la información cuantitativa se realizó mediante la toma general de 40 encuestas a diversas personas del sector por parte de los estudiantes y profesores de la Escuela de Ingeniería Automotriz de la Universidad Internacional del Ecuador. El trabajo de campo se basó en recolectar los datos y posteriormente analizar los resultados de las encuestas, las mismas que tuvieron un carácter descriptivo no inferencial, con preguntas orientadas a obtener resultados que permitieron tabular y analizar las características generales y coincidentes de las personas encuestadas. Las preguntas más representativas estuvieron dirigidas a indagar sobre condiciones del sector como el nivel de señalización, accidentes (fuentes y concentración geográfica), conocimientos de leyes de tránsito, alcantarillado y servicios básicos. Esta exploración de datos se realizó a una muestra no representativa para la población del sector. Las estadísticas resultantes de este trabajo investigativo están estudiadas en las tablas dinámicas de Excel que se presentan posteriormente.

\section{Resultados}

Con la tabulación de los datos obtenidos en las encuestas que fueron realizadas en el sector de Chongón, se presenta la información de los habitantes relacionada con accidentes y señalización, así como servicios básicos y alcantarillado. 


\section{Tabla 1}

Percepción del nivel de accidentes en Chongón

\begin{tabular}{|c|c|c|}
\hline & No. Personas & Porcentaje \\
\hline Alto (3) & 10 & $25 \%$ \\
\hline Medio (2) & 21 & $53 \%$ \\
\hline Bajo (1) & 9 & $22 \%$ \\
\hline Total & 40 & $100 \%$ \\
\hline
\end{tabular}

\section{Tabla 2}

Percepción de la señalización vial en Chongón

\begin{tabular}{lccc}
\hline & & No. Personas & Porcentaje \\
\hline Suficiente & $(3)$ & 3 & $8 \%$ \\
Regular & $(2)$ & 21 & $52 \%$ \\
Insuficiente & $(1)$ & 16 & $40 \%$ \\
\hline \multicolumn{2}{l}{ Total } & $\mathbf{4 0}$ & $\mathbf{1 0 0 \%}$
\end{tabular}

Nota: Esta tabla muestra una percepción de regular a insuficiente por el nivel de señalización vial en el sector de Chongón de acuerdo a resultados de una encuesta realizada a habitantes de la zona.

Como se presenta en la tabla 1, de acuerdo a la información obtenida en las encuestas, los habitantes del sector de Chongón consideran que existe una alta probabilidad de accidentes de vehículos ( $78 \%$ considerando nivel medio y alto), mientras que en la tabla 2, los resultados de las encuestas permiten conocer que los habitantes tienen una percepción de señalización insuficiente $(40 \%)$.

\section{Tabla 3}

Fuente de accidentes

\begin{tabular}{lcc} 
& No. Personas & Porcentaje \\
\hline Motos & 28 & $70 \%$ \\
Tricimotos & 5 & $12 \%$ \\
Particulares & 4 & $10 \%$ \\
Buses & 3 & $8 \%$ \\
\hline Total & $\mathbf{4 0}$ & $\mathbf{1 0 0 \%}$ \\
\hline
\end{tabular}

Nota: Esta tabla muestra la percepción del origen de los accidentes en el sector de Chongón de acuerdo a resultados de una encuesta realizada a habitantes de la zona, donde se pudo observar una gran cantidad de motos y tricimotos como medio de transporte y justamente estos concentran la mayor parte de las fuentes de accidentes. 


\section{Tabla 4}

Concentración de accidentes

\begin{tabular}{lcc} 
& No. Personas & Porcentaje \\
\hline Centro de Chongón & 25 & $62 \%$ \\
Km 24 Vía a la Costa & 7 & $18 \%$ \\
(Ingreso a Chongón) & 8 & $20 \%$ \\
Otros sectores & $\mathbf{4 0}$ & $\mathbf{1 0 0 \%}$ \\
\hline Total & & \\
\hline
\end{tabular}

Nota: Esta tabla muestra la percepción de la concentración de los accidentes que se dan en el sector de Chongón de acuerdo a resultados de una encuesta realizada a habitantes de la zona donde se tiene una alta concentración de accidentes en el centro de Chongón.

En las tablas 3 y 4 se detallan las fuentes de accidentes y la concentración de los mismos. Las motos son la principal fuente de accidentes (70\%) y estos ocurren con mayor frecuencia en el centro de Chongón (62\%), de acuerdo a la información obtenida en las encuestas.

\section{Tabla 5}

Servicios Básicos en Vivienda

\begin{tabular}{lcc}
\hline & No. Personas & Porcentaje \\
\hline Si & 30 & $75 \%$ \\
No & 10 & $25 \%$ \\
\hline Total & $\mathbf{4 0}$ & $\mathbf{1 0 0 \%}$ \\
\hline
\end{tabular}

Nota: Esta tabla muestra los resultados de una encuesta donde se consultó si posee servicios básicos a habitantes del sector de Chongón, pudiendo apreciar que existen deficiencias en el sector.

\section{Tabla 6}

Nivel del Servicio de Alcantarillado

\begin{tabular}{lcc} 
& No. Personas & Porcentaje \\
\hline Insuficiente & 13 & $32 \%$ \\
Regular & 19 & $48 \%$ \\
Suficiente & 8 & $20 \%$ \\
\hline Total & $\mathbf{4 0}$ & $\mathbf{1 0 0 \%}$ \\
\hline
\end{tabular}

Nota: Esta tabla muestra una percepción de regular a insuficiente sobre el nivel del servicio de alcantarillado en el sector de Chongón de acuerdo a resultados de una encuesta realizada a habitantes de la zona.

La tabla 5 permite conocer que el $75 \%$ de los encuestados posee servicios básicos, sin embargo, en la tabla 6 se puede ver que la opinión de estos sobre el nivel del servicio de alcantarillado en el sector se inclina hacia regular (48\%) e insuficiente (32\%). 


\section{Tabla 7}

Linea Base

\begin{tabular}{|c|c|}
\hline Categoría & Resultado \\
\hline Género & $48 \%$ de los encuestados son hombres y $53 \%$ mujeres. \\
\hline Edad & El promedio de edad de los encuestados fue de 34 años. \\
\hline Estado Civil & El $50 \%$ de los encuestados son solteros, $35 \%$ casados y el $15 \%$ no fue definido. \\
\hline Hijos & De la encuesta el $40 \%$ de las personas tienen hijos y el $60 \%$ no. \\
\hline Formación & El 57\% tiene educación secundaria y el $25 \%$ educación superior. \\
\hline Vehículo & El $67 \%$ no cuenta con vehículo propio. \\
\hline Licencia & El 33\% cuenta con un tipo de licencia de conducir. \\
\hline $\begin{array}{l}\text { Manejo } \\
\text { Residuos }\end{array}$ & El 33\% señala que no tiene conocimientos sobre el manejo de residuos. \\
\hline Ley de tránsito & El 58\% indica que no tiene conocimientos de leyes de tránsito. \\
\hline Capacitación & El 93\% señala que está interesado en recibir capacitación. \\
\hline $\begin{array}{l}\text { Disponibilidad } \\
\text { por } \\
\text { capacitación }\end{array}$ & $\begin{array}{l}\text { El } 55 \% \text { de los encuestados podría recibir capacitación los fines de semana por la } \\
\text { mañana. }\end{array}$ \\
\hline \multirow{4}{*}{$\begin{array}{l}\text { Temas de } \\
\text { interés por } \\
\text { capacitación. }\end{array}$} & $45 \%$ en requisitos y formación teórica para obtener licencia tipo A \\
\hline & $28 \%$ en educación vial orientado en prevención de accidentes \\
\hline & $14 \%$ en mantenimiento preventivo de motos y tricimotos \\
\hline & $13 \%$ seguridad industrial enfocado en el manejo de residuos \\
\hline
\end{tabular}

Nota: Se puede observar que el alto porcentaje de desconocimiento en leyes de transito se puede relacionar con el interés en capacitación en educación vial.

Esta tabla ha sido adaptada de "Determinación de la línea base del programa de vinculación de la Universidad Internacional del Ecuador extensión Guayaquil”, Izaguirre, J., Bazurto, E., Rosado, P., 2020, Innova, 5(2), 133-139. doi: 10.33890/innova.v5.n2.2020.1277

En la tabla 7 se presentan los resultados que marcan la línea base para la investigación obtenidos en el levantamiento de información en el sector de Chongón. Con los resultados detallados se busca potenciar los puntos débiles en temas viales y ambientales de la zona.

\section{Conclusiones}

El presente documento tuvo como objetivo analizar la percepción de habitantes del sector de Chongón con un enfoque vial y ambiental, para así poder elaborar un proyecto de vinculación para la Escuela de Ingeniería Automotriz de la Universidad Internacional del Ecuador extensión Guayaquil. En este estudio se procedió a llevar a cabo una investigación cuantitativa mediante el uso de encuestas como herramienta. Mediante los datos de la muestra no representativa se obtuvo información que permite conocer más el entorno de las personas con las que se desean desarrollar actividades comunitarias y definir una línea base de investigación. Frente a esto, se podrán elaborar 
actividades enfocadas a incrementar conocimiento en educación vial y ambiental en habitantes de la zona.

En la investigación se evidenció la necesidad de realizar actividades en temas relacionados a educación vial y gestión ambiental tanto en habitantes como negocios del giro automotriz del sector. Las medidas propuestas están basadas en los resultados obtenidos correspondientes al transporte mediante motos y tricimotos, así como nivel regular-insuficiente de alcantarillado, debido a que son los más podrían generar impacto para evitar accidentes de tránsito en el centro de Chongón y contaminación por residuos que surgen del mantenimiento particular que pueden realizar los conductores. Por lo tanto, dentro de las primeras actividades de vinculación necesarias se encuentra la ejecución de actividades con un enfoque integral que atienda tanto a peatones como conductores para incrementar el conocimiento en educación vial y negocios del giro automotriz que brinden servicios de mantenimiento para dar a conocer la correcta gestión de residuos.

Además, es fundamental considerar la necesidad de impartir capacitaciones en educación vial orientada en prevención de accidentes, así como requisitos y responsabilidades para la obtención de licencias tipo A, con el propósito de que los habitantes reconozcan el impacto que estas medidas tendrían en los accidentes que se generan en la zona, para así incrementar su conocimiento y capacidad de reacción ante una situación de riesgo. Asimismo, es importante desarrollar charlas y capacitaciones dirigidas a conductores de motos, cooperativas de tricimotos y talleres con el objetivo de generar conciencia en temas ambientales, así como dar a conocer normativas y planes de gestión de residuos que pueden surgir como resultado del mantenimiento de medios de transporte, esto debido la falta de infraestructura en el servicio de alcantarillado que aumenta el riesgo de contaminación por lubricantes, combustibles y otros fluidos peligrosos.

Para finalizar, considerando el incremento acelerado en ventas de motos en los últimos años y proyectos habitacionales en la zona, se concibe que los niveles de accidentes, así como la contaminación pueden incrementarse. La implementación de proyectos de capacitación se podría generar transferencia de conocimiento en la población del sector a medida que se da el incremento personas y parque automotor por proyectos habitacionales entregados en Chongón.

\section{Referencias Bibliográficas}

Asamblea Nacional República del Ecuador. (2018). Ley Orgánica Reformatoria a la Ley Orgánica de Educación Superior. Quito, Ecuador.

Asociación de Empresas Automotrices del Ecuador. (2019). Anuario 2019. (A. d. Ecuador, Ed.) $\begin{array}{llllll}\text { Recuperado el } 10 \quad \text { de } & \text { septiembre } & \text { de }\end{array}$ https://www.aeade.net/sdm_downloads/anuario-2019/

Autoridad Aeroportuaria de Guayaquil. (02 de diciembre de 2015). Futura Ciudad Aeroportuaria Chongón Daular. Recuperado el 11 de septiembre de 2020, de http://www.aag.org.ec/index.php/projects/255-aeropuerto-de-guayaquil

Bastidas, P. \& Velasco, E. (2018). Plan de Desarrollo Turístico para la Comuna San Pedro de Chongón como complemento a la oferta turística de Guayaquil. Guayaquil, Ecuador: 
Universidad Católica de Santiago de Guayaquil. Obtenido de http://repositorio.ucsg.edu.ec/handle/3317/10243

Carhuancho, I., Sicheri, L., Nolazcos, F., Guerrero, M., Casana, K. (2019). Metodología de la investigación holística (Vol. 1). Guayaquil, Ecuador: UIDE. Obtenido de https://repositorio.uide.edu.ec/handle/37000/3893

Diario Expreso. (8 de junio de 2019). Autoridad: 65\% de Chongón tendrá alcantarillado este año.

Guerrero, M., Cava, J., Villanueva, A., Hernández, R., Fernández, J., \& Berbel, J. (2019). Fomentando la vinculación entre empresa e invsestigación en el Grado en Administración y Dirección de Empresas. Revista de innovación y buenas prácticas docentes.

Hurtado de Barrera, J. (2015). El proyecto de investigación, comprensión holística (Vol. 8va). Caracas: Sypal.

Instituto Nacional de Estadísticas y Censos. (2018). Anuario de Estadística de Transporte (ANET). $\begin{array}{llllll}\text { Recuperado el } 10 \text { de } & \text { septiembre }\end{array}$ https://www.ecuadorencifras.gob.ec/transporte/

Instituto Nacional de Estadísticas y Censos. (2019). Encuesta Nacional de Empleo, Desempleo y Subempleo (ENEMDU). Recuperado el 11 de septiembre de 2020, de https://www.ecuadorencifras.gob.ec/documentos/webinec/EMPLEO/2019/Septiembre/Boletin_tecnico_de_empleo_sep19.pdf

Interagua. (s.f.). Ajuste y revisión del plan maestro agua potable; alcantarillado sanitario y alcantarillado pluvial (Vol. Tomo II). Guayaquil, Guayas, Ecuador. Recuperado el 10 de 2020, de https://www.interagua.com.ec/sites/default/files/portal-de-transparencia/planesprogramas/tomo_ii.pdf

Izaguirre, J., Bazurto, E., Rosado, P. (2020). Determinación de la línea base del programa de vinculación de la Universidad Internacional del Ecuador extensión Guayaquil. Innova, 5(2), 133-139. https://doi.org/10.33890/innova.v5.n2.2020.1277

Loaiza, C. \& Gómez, E. (2019). Estudio y estimación de emisiones generadas por motos. $\begin{array}{llllll}\text { Recuperado el } 10 \text { de } & \text { septiembre }\end{array}$ https://repositorio.uide.edu.ec/handle/37000/3986

Martínez, M. (2007). La investigación cualitativa etnográfica. México: Trillas.

Mite, S. \& Reyes, S. (2018). Estudio socioeconómico de la parroquia San Jerónimo de Chongón para impulsar su desarrollo local. Guayaquil, Ecuador: Universidad de Guayaquil Facultad de Ciencias Administrativas. Obtenido de http://repositorio.ug.edu.ec/handle/redug/27400 